\title{
Application of the Markov Chain Theory in Estimating the Strength of Fiber-Layered Composite Structures with Regard to Manufacturing Aspects
}

\author{
Rafał Chatys ${ }^{1 *}$ \\ 1 Kielce University of Technology in Kielce, Faculty of Mechatronics and Machine Building, AL.1000-lecia P.P. 7, \\ 25-314, Kielce, Poland \\ *Corresponding author's e-mail: chatys@tu.kielce.pl
}

\begin{abstract}
The following work describes the application of a mathematical model based on Markov chain theory (MM) to estimate the fiber composite strength parameters, taking into account the laminate forming aspects using the vacuum bag method. This provides, from a unified position, a tool for a coordinated description of static strength and fatigue life. It was shown that the MM model gives relatively good results.
\end{abstract}

Keywords: composite, fiber, fatigue strength, technological aspects, Markov theory

\section{INTRODUCTION}

The industry needs algorithms, analytical methods confirmed by knowledge and information when verifying the strength properties of fibrous composite materials (FCM), which are increasingly common $[2,14,22,23,26]$.

Estimating the mechanical properties of polymer matrix composite components (fibers, fiber bundles, or matrix) is a difficult task. The verification of most numerical models of laminates takes into account only the initial stages of the complexity of polymer structures. Determining the fatigue life of the components or elements made of FCM after a certain period of variable random loads is a much greater challenge [39]. In the literature, the works of Cullmann [12], Iosifescu [19], Fleming and Soner [15], Dynkin, Liggett and White [40] reflect the proposals of random processes through mathematical models. Despite this, the calculations do not allow stating unequivocally which of the current models accurately describes the development and course of damage by changing the value of mechanical properties taking into account material constants $[4,28-30,36]$.
One of the variants of describing the relationships characterizing damage accumulation or fatigue summation $[3,20,25,27,38,42]$ are phenomenological hypotheses of fatigue damage accumulation [31, 32, 35] and Markov network $[5,13,41]$. However, this is not a new idea [21]. In the proposed modification $[6,33,34]$ of the Markov model (MM) as a class of stochastic processes, it uses discrete observations (describing the path when the parameter is continuous) and finite Markov chains (probability theory). The proposed relationships (distributions) of static and fatigue strength are based on a random combination of $n$ elements that are fit or unfit, with the possibility of a sharp increase in the number of states (and, thus, transitions and the number of system elements).

\section{MODEL}

The destruction of a certain critical micro volume (CMV) in a material consisting of longitudinal reinforcement (in the form of fibers or bundles of fibers working in the elastic range - rigid) and a plastic matrix (in which plastic deformations 
accumulate during cyclic loading) determines the fatigue damage of the composite.

In addition, the model assumes that the CMV elements (including the following components: the matrix and other fiber layers with a different angle than the fibers working along the load) work together.

This means that before the plastic deformation of a sample part reaches the value of $\varepsilon_{\mathrm{Y}}$ (accumulation of irreversible plastic deformations), the fibers and the matrix work together in the elastic range. Therefore, as a result of cyclical load rearrangement, the number of working elements $r$ in CMV (in the elastic range) decreases by the value of $r_{R}$ (until the sample is slowly destroyed).

Fatigue strength is described by a matrix of probability transitions (1), in which all probabilities below the diagonal are equal to zero. If, in addition, the passages to the first level are allowed in the process (and there is only one absolute state), then the damage accumulation will be modelled first (Markov chain). In many phenomenological models, the problem of discussing the basics, ideas and methods of stochastic modeling of the fatigue process is raised and investigated $[6,13$, $16,33,41]$. Selected probabilities can create conditional probabilities.

$$
P=\left[\begin{array}{ccccccc}
q_{1} & p_{1} & 0 & & & \ldots & 0 \\
0 & q_{2} & p_{2} & 0 & & \ldots & 0 \\
0 & 0 & q_{3} & p_{3} & 0 & \ldots & 0 \\
\ldots & \ldots & \ldots & \ldots & \ldots & \ldots & \ldots \\
0 & & & \ldots & q_{r} & p_{r} & 0 \\
0 & & & \ldots & 0 & 0 & 1
\end{array}\right]
$$

where: $q_{i}=1-p_{i}, i=1, \ldots, r$

Then, the random variable generates the probabilities of the variable $\mathrm{T}$ (i.e. the inverse transformation) determines the distribution of this probability by the relationship (6) and (7) respectively, with the cumulative distribution function (8). The parameters of the Markov chain are presented in Table 1.

It needs to be considered that the product of the matrix $P^{i}$ (9) times vector $\mathrm{b}$ gives the column vector of the fatigue strength distribution function, the elements of which correspond to the initial (start) states of the Markov chain: $\left(F_{T}^{(1)}(t) F_{T}^{(2)}(t), \ldots . F_{T}^{(r)}(t)\right.$. In the general case, it can be used to determine the fatigue strength

Table 1. Characteristics of the Markov chain $[6,7]$

\begin{tabular}{|c|c|c|}
\hline Characteristics & Dependencies & \\
\hline Fatigue strength (time to absorption) & $\begin{array}{c}\quad T=X_{1}+X_{2}+\ldots+X_{r} \\
\text { where: } X_{i}, i=1 ; r-\text { time of destruction (is) in an } i-m \text { state. }\end{array}$ & (2) \\
\hline Random variable $X_{i}$ in a geometric distribution & $P\left(X_{i}=n\right)=\left(1-p_{i}\right)^{n-1} p_{i}$ & (3) \\
\hline Expected value & $E\left(X_{i}\right)=1 / p_{i}$ & (4) \\
\hline Dispersion & $V\left(X_{i}\right)=\left(1-p_{i}\right) / p_{i}^{2}$ & (5) \\
\hline Random variable & $E(T)=\sum_{i=1}^{r} 1 / p_{i} \quad V(T)=\sum_{i=1}^{r}\left(1-p_{i}\right) / p_{i}^{2}$ & (6) \\
\hline $\begin{array}{l}\text { A function that generates the probabilities of a random } \\
\text { variable } T\end{array}$ & $G_{T}(z)=\sum_{i=0}^{\infty} p_{T}(i) \cdot z^{i} \prod_{i=1}^{\infty} \frac{z p_{i}}{1-z\left(1-p_{i}\right)}$ & (7) \\
\hline Cumulative distribution function & $\begin{array}{l}F_{T}(t)=p_{1 r+1}(t), \mathrm{t}=1,2,3 \\
\text { where: } p_{1 r+1}(t) \text { is }(1, \mathrm{r}+1)-\text { matrix element }\end{array}$ & (8) \\
\hline Fatigue strength distribution function & $\begin{array}{l}\qquad P(t)=P^{t} \\
\text { described as: } \\
F_{T}(t)=a P^{t} b \\
\text { where: } a=(100 \ldots 0) ; b=(00 \ldots 01) T-\text { column vector }\end{array}$ & (9) \\
\hline
\end{tabular}


distribution function $F_{T}(t)$, with a given probability distribution under starting conditions $\mathrm{p}$ :

$$
F_{T}(t)=\pi P^{t} b, \text { (with knows } \pi \text { ) }
$$

In the application of the problem under consideration, the problem consists in finding the relationships between the probabilities, $p_{i}, \mathrm{i}=1, \ldots, r$ with the values of the distribution of the static strength of the FCM elements and fatigue loads, respectively. It is assumed that in the first step of the Markov chain (e.g. 1 or 1000 cycles) it destroys one element.

If working parallel elements (R-i) in the laminate have the same static strength distribution function $\mathrm{F}(\mathrm{s})$ with the expected probability (11), then with a given distribution (e.g. log-normal distribution F (s): where: is $\Phi($.$) a function of$ the standard normal decomposition), the destruction occurs:

$$
p_{i}=1-\left(1-F\left(s_{i}\right)\right)^{R-i}
$$

where: $s_{\mathrm{i}}-$ stress (load on one element), corresponding to the uniform distribution of the load among other (R-i) elements;

$R$ - initial number of elements in the "critical volume" of the composite, the failure of which represents the complete failure of the specimen;

$i$ - number of elements destroyed; log-normal strength distribution: $F(s)=\Phi\left(\left(g(s)-\theta_{0}\right) / \theta_{1}\right)$, where $\theta_{0}, \theta_{1}$ - parameters of the static strength distribution of composite elements (expected value and standard deviation of strength on a logarithmic scale).

The considered model is determined in the general case with the constant $\eta=\left(\theta_{0}, \theta_{1}, r, R, k_{M}, S_{f}\right)$, having 6 components, where: $\mathrm{r}$ - critical number of elements in FCM ( $\mathrm{r}$ value has a significant impact on dissipation and fatigue strength variation coefficient); relationship $\rho=r / R$ approximately determines the value of the destruction (contribution of destruction) in the cross section of the sample corresponding to the total destruction of the sample; $\mathrm{k}_{\mathrm{M}}$ - number of cycles corresponding to one step of the Markov chain.

\section{Methodology of forming a composite. Statistical and fatigue tests}

In order to carry out the task, the components (glass fabric $\left[0^{\circ} / 90^{\circ}\right]$ and carbon fabric [11] of the biaxial type $\left[+45^{\circ} / 45^{\circ}\right]$ with a basis weight 600 and $400 \mathrm{~g} / \mathrm{m}^{2}$ respectively, and epoxy resin LH-160 [10]) of composite molding using the vacuum bag method were selected [17]. With the resulting vacuum in the vacuum method and the adopted assumptions (normal atmospheric pressure $1013 \mathrm{hPa}$ and gravitational acceleration of $9.8145 \mathrm{~m} / \mathrm{s}^{2}$, for different levels of vacuum pressure per unit area [24]) resulting from the intensity of resin crosslinking, determining the proportion of volume and properties of hardened resin [19] associated with the resin flow resistance is a quite significant problem.

The flow of resin mixture and heat exchange in a layer package (layer system - preform) with constant porosity is two-dimensional. Assuming this flow as a flow of viscous liquid with the relationships between pressure, viscosity and speed occurring in it, its movement can be described by the Poiseuille'a relationship over a specific period of time:

$$
\Delta p=\frac{32 \eta L u}{d^{2}}
$$

where: $\Delta p$ - pressure drop caused by fluid flow viscosity resistance;

$\eta$ - dynamic fluid viscosity;

$L$ - pipe length;

$u$ - fluid flow rate;

$d$-pipe diameter.

Considering this problem from the point of view of the Darcy law (24), the area at the edge will be the channel for supplying the resinous medium with a much larger diameter of the crosssection A (Fig. 1).

If we consider the capillary surface of a preform with unlimited permeability, then when sucking (soaking) the resin mixture, the volume of the preform (proportion of resin volume to

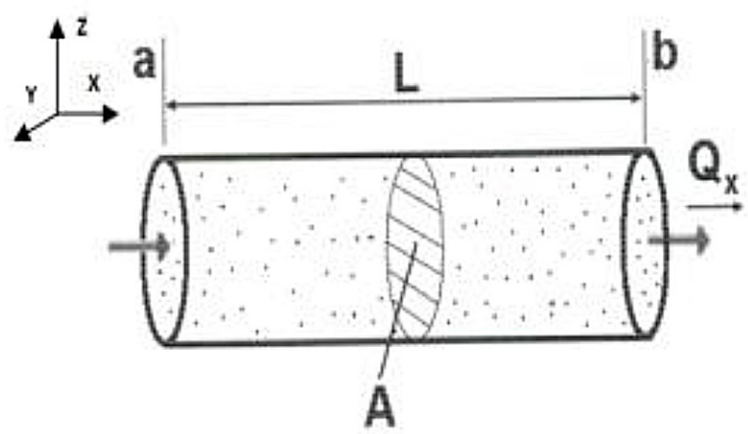

Fig. 1. Schematic of the simplified model along coordinate system 
strengthen) changes accordingly and the pressure dropping in the preform over time is observed (caused by local resistance, different temperature and, thus, the viscosity of the mixture resin).

Reducing the flow resistance of liquid resin at the edges of the preform changes the pressure conditions in the mold, disrupting the saturation process according to the Darcy-Weisbach formula (13).

$$
\Delta h_{m}=\zeta \frac{u_{a}^{2}}{2 g}
$$

where: $\Delta \mathrm{h}_{\mathrm{m}}$ - height of local piezometric pressure losses;

$\zeta$ - coefficient of local resistance (linear friction resistance);

$u_{a}$ - average flow velocity in cross section, in section or in form; $\mathrm{g}$ - acceleration due to gravity.

Due to the thermal dispersion, which is the difference between the total microscopic and average heat convection (as a result of gelation) determined with a specific heat conduction coefficient (as a diffusion process), we can refer to the total temperature gradient, which (for the patency of the fibrous medium) is described by the Carman-Kozeny model [9].

It is important to establish a safe distance (i.e. $2 / 3$ of the maximum distance) of suction from the mold (flow) of the resin medium with the hardener [18], remembering not to create a branch coming down from the saturated / suction reinforcement (which the resin mixture may encounter in the vacuum created).

The lack of accurately estimated distances may result in sealing the dry reinforcement surfaces, in which air will be trapped instead of the resinous medium. It is also worth mentioning that the resin medium is not crosslinked [24] as a result of fewer crosslinks created by styrene during the rapid gelation of the mass (or after curing time). All observations and corrected errors should be introduced in subsequent processes and included in the technological data sheets [8].

The very process of FCM molding with the method of "pressing the resin under pressure in closed molds" is not simple, but with adequate experience it is predictable. In this vacuum bag method, special attention should be paid to the first layer of reinforcement, which determines the smoothness and quality of the obtained laminate composite surface (and the separation of the produced laminate from the mold after curing).

Therefore, before soaking the resin with reinforcement, it is important to prepare the surface of the mold by applying a release agent (wax) and polishing. Then, to ensure the tightness of the system and maintain an even vacuum during the whole process, a double-sided tape is applied on the edges of the mold (to which the flexible foil will be glued). Then, the first layer of epoxy resin (LH 160) with the hardener (L 289) is made with the recommended weight ratio and 3 layers of reinforcement: carbon fabric, glass mat and carbon fabric on the outside. In addition, the preform laid in the laminate is secured with auxiliary materials in the form of "peel ply" fabric (so-called delamination), breathable nonwoven fabric and perforated foil. The layer package prepared with the valve attached in this way is used to check the hermeticity of the system.

Then, a 3-layer laminate could be formed under pressure $-0.85 \div-0.9$ bar (in the "Composite Forming" Laboratory at Kielce University of Technology) from which the samples were cut according to PN-EN 10002-1 + ACI (Fig. 2) on the APW2010BB machine (with a stream velocity of $0.8 \mathrm{~m} / \mathrm{s}$ ) for static tensile testing (according to DIN-EN ISO 527).

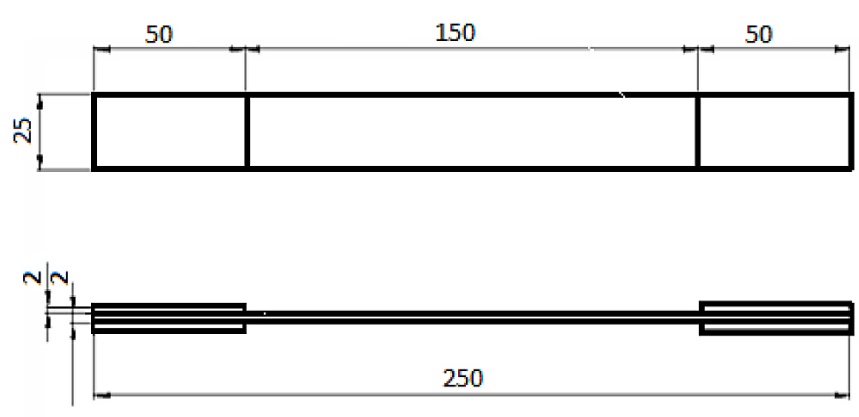

Fig. 2. The geometrical dimensions of the composite samples 
In order to reduce the impact of stress concentration on the mechanical properties of the composite before surface destruction, the pads were glued to the samples (thus, increasing the area of even distribution of stress concentration in the tested sample).

In order to eliminate the surface damage of the sample (thus, increasing the surface of a uniform distribution of stress concentration in the tested sample), overlays were glued to the samples.

The static tensile test for the samples prepared in this way was carried out on an INSTRON testing machine equipped with a Flex Test SE measuring apparatus and an MTS controller [1]. The loads were measured using the HBM 1-XY91-6 / 350 rosettes which included two perpendicular strain gauges and a single HBM 1-XY91-6/350 meter (with the same measuring lengths of $6 \mathrm{~mm}$ and nominal electrical resistance of $350 \Omega$ ). The tensile tests were performed with the machine head moving speed of $2 \mathrm{~mm} / \mathrm{min}$. Measuring the ratio of force to the measured mean cross-sectional area of the test specimens enabled to estimate the (axial) stress. Poisson's ratio (vxy), the transverse deformation - axial deformation was determined from the curve. The determination of the average value of the static strength $\left(\mathrm{S}_{\text {stati }}\right)$ of the laminate was used to determine the fatigue curve $(\mathrm{S}-\mathrm{N})$.

The tests were carried out with the maximum number of cycles (up to 35.000) at fixed strength levels. The samples from the epoxy-based polymer composites were subjected to cyclic loading on 3 levels $\mathrm{K}^{*} \mathrm{~S}_{\text {statist }}(\mathrm{K}=0.3 ; 0.4 ; 0.5)$, with the assumed frequency $f=6 \mathrm{~Hz}$.

\section{ANALYSIS OF RESULTS}

The destruction of the 3-layer laminate took place by cutting the interface between the phase and the resin layer between the two carbon layers, in which there was a layer of glass matotextile (caused by defects in the structure of the composite produced, and sample cutting parameters). The determined value of the average strength of five samples was estimated at $311.6 \mathrm{MPa}$ (i.e. up to $10 \%$ deviation from the average value) as a result of the change in the volume of resin medium to the reinforcement (Table 2) and the pressure drop in the bag over the suction time (caused by local resistance, temperature gradient, and thus the viscosity of the resin mixture). The accumulation of defects and damage (microcracks, microcracks in components or voids in the epoxy matrix) in the composite structure deteriorate the mechanical properties of the produced laminate.

Debonding as a microstructural damage that causes a loss of adhesion at the interface between the components (reinforcement with the matrix) is visible in the early stages of the degradation process of polymer composites. At this stage of material destruction, the adhesive cracks do not have a significant impact on the macroscopic characteristics of the material yet, but are usually the first link in the material destruction chain. The damage to the boundary layer occurs as a result of exceeding the values of critical stresses, i.e. stresses normal to the side surface of the fiber, and tangential stresses (shear of the fiber-matrix contact zone).

\section{MODELLING}

The known values of the model (formulas 5, $7,9)$ allow determining the fatigue curve assuming that one step in the Markov chain corresponds to $\mathrm{k}_{\mathrm{M}}$ cycles (Table 3), taking into account the modified formula 6 (Table 1). The modeling of the average fatigue strength $\mathrm{E}(\mathrm{T}(\mathrm{S}))$ at any stress $S$ allows the use of a model with a stationary Markov chain when recreating the fatigue curve $[6$, 37]. Good fitting of the fatigue curve (T-N) data to the experimental results at three stress levels $\mathrm{K} \cdot \mathrm{S}_{\text {statist }}\left(\mathrm{K}_{0.1}=0.3 ; 0.4 ; 0.5\right)$ with a $50 \%$ (Fig. 3a)

Table 2. Mechanical properties of composite A made using the vacuum bag method

\begin{tabular}{|c|c|c|c|c|}
\hline Sample & $\mathrm{F}_{\max }, \mathrm{N}$ & $\varepsilon, \mathrm{mm}$ & $\mathrm{S}_{\max }, \mathrm{MPa}$ & $\mathrm{E}, \mathrm{MPa}$ \\
\hline A1 & 1137.5 & 1.24 & 303.0 & 4.9 \\
\hline A2 & 1047.0 & 1.20 & 3.1 & 5.0 \\
\hline A3 & 1226.0 & 1.38 & 327.0 & 4.9 \\
\hline A4 & 1148.0 & 1.18 & 306.0 & 4.7 \\
\hline A5 & 1287.0 & 1.44 & 343.0 & 4.92 \\
\hline
\end{tabular}


Table 3. Model parameters when fitting the data of T-N curves for sandwich composites with different share of reinforcement $(\mathrm{R}=0.1)$

\begin{tabular}{|l|c|}
\hline \multicolumn{1}{|c|}{ Parameter } & $\begin{array}{c}\text { Parameter values for laminate with 50 (40)\% } \\
\text { reinforcement }\end{array}$ \\
\hline Average value of longitudinal elements strength, $\left(\mathrm{L}_{\mathrm{R}}\right)^{*}$ & $5.742(5.692)$ \\
\hline $\mathrm{R}=\exp \left(\mathrm{L}_{\mathrm{R}}\right), \mathrm{MPa}$ & $311.6(296.5)$ \\
\hline $\begin{array}{l}\text { Mean standard deviation }\left(\mathrm{Std}_{\mathrm{RP}}\right) \text { strength of longitudinal elements on a } \\
\text { logarithmic scale }\end{array}$ & 0.1 \\
\hline The number of longitudinal elements in the critical micro volume $\left(\mathrm{r}_{\mathrm{RP}}\right)$ & 10 \\
\hline $\begin{array}{l}\text { The relative value of the surface area of working longitudinal elements } \\
\text { in FCM }\left(\mathrm{f}_{\mathrm{RO}}\right)\end{array}$ & $0.40(0.50)$ \\
\hline Number of cycles equivalent to one step in the Markov chain $\left(\mathrm{k}_{\mathrm{m}}\right)$ & $9505(5402)$ \\
\hline
\end{tabular}

* Natural logarithm calculations.

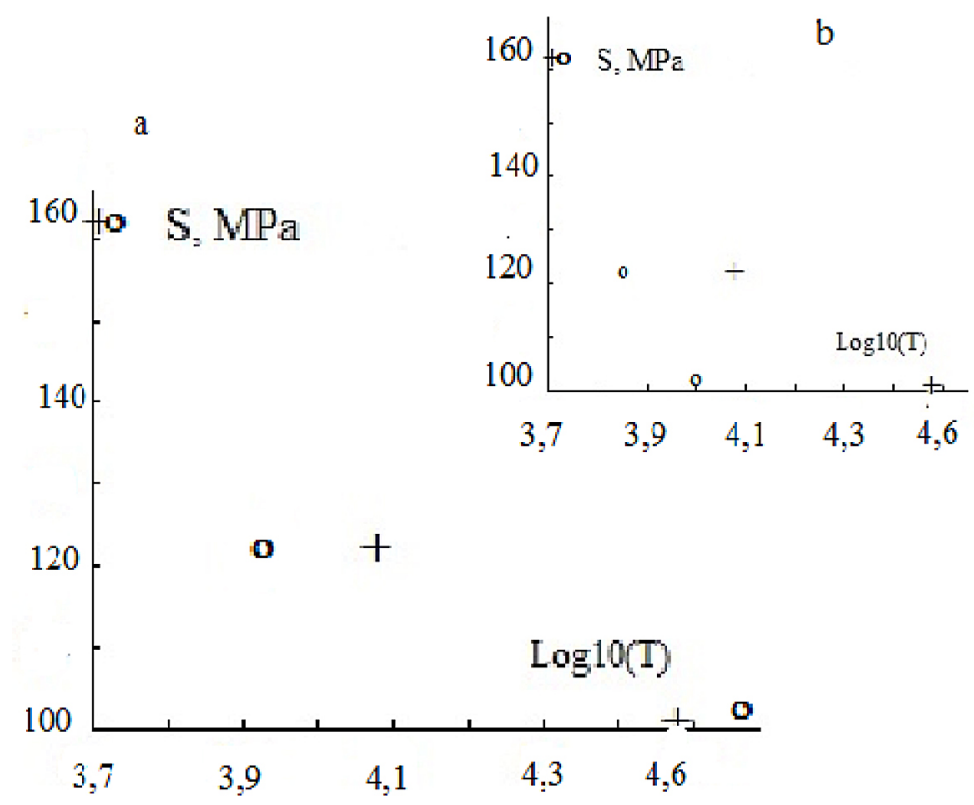

Fig. 3. Experimental $(+)$ and estimated (o) average fatigue strength for three loading values for fibrous composite A molded in vacuum bag $\left(S_{l}, n_{l}\right)=(108,85 \mathrm{MPa} 35000 ; 124,40 \mathrm{MPa}$,

$20000 ; 155,50 \mathrm{MPa}, 3300$ ) with a gain of 50 (a) and 40 (b)\% of reinforcement share

share of the reinforcement quite well describe the "upper" and "lower part"of the laminate fatigue curve $(\mathrm{R}=0.1)$. On the other hand, the adjustment of the T-N curve data for a sandwich composite with a $40 \%$ reinforcement share (Fig. 3b) shows a more significant dispersion of the "lower" and "average part" of the T-N curve, than it was for a laminate with a $50 \%$ reinforcement share.

\section{CONCLUSIONS}

The analysis of the obtained calculations shows that the considered modeled data of the epoxy matrix composite with a stationary Markov chain model describe the "upper part" of the fatigue curve and the "lower part" for the composite with a $50 \%$ strengthening share fairly well. The model with the analysis of the statistical parameters can be a tool for "predicting" the S-N curve, taking into account not only the maximum volume share of reinforcement in the composite, but also the manufacturing technology.

\section{REFERENCES}

1. ASTM D638 - Standard Test Method for Tensile Properties of Plastics.

2. Behm G.W, Huber W.B, Noll A.J, Pelaez R. A Method and system for safe emergency vehicle operation using route calculation. United States Patent US8842021B2. 2014.

3. Blassiau, S., Thionnet, A., Bunsell, A.R. Micromechanics of Load Transfer in a Unidirectional Carbon Fiber-Reinforced Epoxy Composite Due to 
Fiber Failures - Part I: Micromechanisms and 3D Analysis of Load Transfer: The Elastic Case. Composite Structures. 74(3), 2006, 303-318. https:// doi.org/10.1016/j.compstruct.2005.04.013

4. Chatys R. Mechanical Properties of Polymer Composites Produced by Resin Injection Molding for Applications Under Increased Demands for Quality and Repeatability. Ultrasound, 64(2), 2009, $35-38$.

5. Chatys R, Paramonova A.Yu., Kleinhof M.A. Analysis of Residual Strength afterFatigue in Fibrous Composite using Markov Chains Mode, Monography: "Selected Problems of Modeling and Control in Mechanics", Edited by St. Adamczak and L. Radziszewski, Kielce, 2011, 166-178.

6. Chatys R. Investigation of the Effect of Distribution of the Static Strength on the Fatigue Failure of a Layered Composite by Using the Markov Chains Theory. Mechanics of Composite Materials, 48(6), 2012, 911-922.

7. Chatys R. Statistical verification of strength parameters of fibrous composite materials. Composites theory and practice, 12(3), 2012, 171-176.

8. Chatys R., Szafran K. Influence of technological aspects of the production of fibrous composite materials during the injection of resin under pressure into the interior of the mold, Transactions of the Institute of Aviation, 3(244), 2016, 41-53.

9. Choi, M. A., Lee, M. H., Chang, J., and Lee, S. J. Permeability modeling of fibrous media in composite processing, Journal of Non-Newtonian Fluid Mechanics, 79, 1998, 585-598.

10. Company directory Havel Composites.

11. Company directory Rymatex.

12. Cullmanna G. Les chaines de Markov multiplles: programmation dynamique. Pris, Masson, 1980.

13. Dekker R, Nicolai R.P, Kallenberg L.C.M, Maintenance and Markov decision models. In Wiley StatsRef: Statistics Reference Online (eds Balakrishnan N, Colton T, Everitt B, Piegorsch W, Ruggeri F, Teugels J.L.). John Wiley \& Sons, 2014, https://doi.org/10.1002/9781118445112.stat03960.

14. Dong W, Liu S, Yang X, Wang H, Fang Z. Balancing reliability and maintenance cost rate of multistate components with fault interval emission. Maintenance and Reliability. 21(1), 2019, 37-45, https://doi.org/10.17531/ein.2019.1.5.

15. Fleming W.H., Soner H.M. Controlled Markov processes and viscosity solutions. New York. Springer Verlag, 1993.

16. Found M.S, Quaresimin M. Two-stage fatigue loading of woven carbon fiber reinforced laminates, Fatigue Fract. Eng. Mater. Struct. 26, 2003, 17-26.

17. http://www.baltazarkompozyty.pl/index. php?option $=$ com_content $\&$ view $=$ article $\& i d=1$ 72:technologia-worka-prozniowego-vacuumbagging \& catid $=15 \&$ Itemid $=46$

18. http://forumwodne.pl/hypeboat-polski-producentszybkich-rowerow-wodnych-t27.html

19. Iscioglu F, Kocak A. Dynamic reliability analysis of a multi-state manufacturing system. Maintenance and Reliability, 21(3), 2019, 451-459, https://doi.org/10.17531/ein.2019.3.11.

20. Kaviany, M. Principles of Heat Transfer in Porous Media, Ed., Springer-Verlag, New York, 1995.

21. Kemeny J.G, Snell. J.L. Finite Marcov Chains, Princeton: N.J. Van Nostrand, 1966.

22. Kłonica M. Comparative analysis of effect of thermal shock on adhesive joint strength!. Advances in Science and Technology Research Journal, 10(32), 2016, 263-268.

23. Kłonica M., Bielawski R. Strength of Joints with "Hi-Lok" Fasteners in Aircraft Safety Considerations. Advances in Science and Technology Research Journal, 13(4), 2019, 87-93.

24. Kozioł M. Vacuum-Presssure-Assisted Impregnation of Stitched and 3D woven Glass-Fiber Preforms, Monograph 644, Publishing Silesian University of Technology, Gliwice, 2016.

25. Kubit A,, Trzepiecinski T, Kłonica M., Hebda M., Pytel M. The influence of temperature gradient thermal shock cycles on the interlaminar shear strength of fibre metal laminate composite determined by the short beam test. Composites Part B, 176, 2019, 107217.

26. Landowski B, Muślewski Ł, Knopik L, Bojar P. Semi-Markov model of quality state changes of a selected transport system. Journal of KONES, 24(4), 2017, 141-148.

27. Lifshitz J.M. Mechanical Behaviour of Engineering Materials. Vol.2 Dynamic Loading and Intelligent Material Systems, Springer-Science + Business Media, B.V., 2001. https://doi.org/ 10.1007/978-94-010-0436-7.

28. Liu Q, Reifsnider KL. Heterogeneous mixtures of elliptical particles: Directly resolving local and global properties and responses Journal of Computational Physics. 235, 2013, 161-181 https://doi. org/10.1016/j.jcp.2012.09.039.

29. Mahieux C.A., Reifsnider K.L. Property Modeling across Transition Temperatures in Polymers, A. Robust Stiffness-Temperature Model. Polymer, 42, 2001, 3281-3291.

30. Mishnaevsky Jr., L., Brondsted, P. Micromechanics of Damage in Unidirectional Fiber Reinforced Composites: 3D Computational Analysis. Comp. Sci. Tech. 69, 2009, 1036-1044.

31. Paramonov Yu., Andersons J. A family of weakest link models for fibre strength distribution. Composites: Part A38, 2007, 1227-1233. 
32. Paramonov Yu.M., Kleinhof M.A., Paramonova A.Yu. Markov Model of Connection Between the Distribution of Static Strength and Fatigue Life of a Fibrous Composite. Mechanics of Composite Materials, 42(5), 2006, 615-630.

33. Paramonov J., Chatys R., Anderson J., Kleinhofs M. Markov Model of Fatigue of a Composite Material with Poisson Process of Defect Initiation, Mechanics of Composite Materials, 48(2), 2012, 211-228.

34. Paramonov J., Kuznetsov A., Kleinhofs M. Realibility of fatigue prone airframes and composite materials, Riga Technical University, 2011.

35. Prusty, B.G., Pan, J.W., Sul, J. Characterization of temperature-dependent behavior of chopped strand mat GRP during low cyclic fatigue, Conference of Composites or Nano Engineering, Honolulu, Hawaii, 2009.

36. Reifsnider KL, Chiu WKS, Brinkman KS, Du Y, Nakajo A, RabbiF., Liu Q. Multiphysics design and development of heterogeneous functional materials for renewable energy devices: The heterofoam story Journal of the Electrochemical Society, 160. 2013, https://doi.org/10.1149/2.012306jes.

37. Reifsnider K.L., Stinchcomb W.W. A Critical-Element Model of the Residual Strength and Life of
Fatigue-Loaded Composite Coupons. Composite Materials: Fatigue and Fracture, ASTM STP 907, pp. 298-313.

38. Sul J.H., Prusty B.G., Pan J.W. A fatigue life prediction model for Chopped Strand Mat GRP at elevated temperatures, Fatig. Fract. Eng. Mater. Struct., 33, 2010, 513-521. https://doi.org/10.1111/ j.1460-2695.2010.01460.x.

39. Szala J. Assessment of fatigue life of machine elements under random load conditions and software. Scientific Notebook. 79, Mechanics 22, Bydgoszcz, 1980.

40. White D.J. Markov decision processes. Chichester: John Wiley, 1992.

41. Wu X, Zou X, Guo X. First passage Markov decision processes with constraints and varying discount factors. Frontiers of Mathematics in China. 10(4), 2015, 1005-1023, https://doi.org/10.1007/ s11464-015-0479-6.

42. Van den Heuvel P.W.J. , Goutianos S., Young, R.J., Peijs T. Failure phenomena in fibre-reinforced composites. Part 6: A finite element study of stress concentrations in unidirectional carbon fibre reinforced epoxy composites Compos. Sci. Technol. 64 (5), 2004, 645-656. 